\title{
Coherence-Assisted Resonance with Sub-Transit-Limited Linewidth
}

\author{
Lei Feng, ${ }^{1}$ Pengxiong Li, ${ }^{1}$ Liang Jiang, ${ }^{2,3}$ Jianming Wen, ${ }^{4}$ and Yanhong Xiao ${ }^{1, *}$ \\ ${ }^{1}$ Department of Physics, State Key Laboratory of Surface Physics, Laboratory of Advanced Materials and Key Laboratory of Micro \\ and Nano Photonic Structures (Ministry of Education), Fudan University, Shanghai 200433, China \\ ${ }^{2}$ Institute for Quantum Information, California Institute of Technology, Pasadena, California 91125, USA \\ ${ }^{3}$ Department of Applied Physics, Yale University, New Haven, Connecticut 06511, USA \\ ${ }^{4}$ Institute for Quantum Information Science, University of Calgary, Calgary, Alberta T2N 1N4, Canada \\ (Received 25 March 2012; revised manuscript received 5 November 2012; published 5 December 2012)
}

\begin{abstract}
We demonstrate a novel approach to obtain a resonance linewidth below the transit limit. The cross correlation between the induced intensity modulation of two lasers coupling the target resonance exhibits a narrow spectrum. $1 / 30$ of the transit-limited width is achieved in a proof-of-principle experiment where two ground states are the target resonance levels. Attainable linewidth is only limited by laser shot noise in principle. The experimental results qualitatively agree with an intuitive analytical model and numerical calculations. This technique can be easily implemented and should be applicable to many atomic, molecular, and solid state spin systems for spectroscopy, metrology, and resonance-based sensing and imaging.
\end{abstract}

DOI: 10.1103/PhysRevLett.109.233006

PACS numbers: 32.70.Jz, 42.50.Gy

Achieving narrow resonance is of long-lasting interest to both fundamental and applied sciences such as spectroscopy, precision measurement, metrology, and sensing. Resonance lines are often broadened, for example, by Doppler effects, inhomogeneous local fields, or power broadening. Techniques to reduce these effects include laser cooling, rephasing via pulse sequences and Ramsey spectroscopy [1], etc. Nonetheless, resonance linewidth is ultimately limited by lifetimes of involved atomic states or the finite interaction time. How to go beyond these limits has been a long-standing important challenge. Existing efforts fall into three categories: (a) coupling the target resonance to a much narrower resonance [2-4], whose width sets the limit of the attainable linewidth, (b) selectively detecting a subset of an atomic ensemble that has a longer-than-average lifetime or transit time [5-10] and (c) using density narrowing [11].

We propose a new approach using continuous-wave lasers and intensity correlations to obtain a linewidth far below the interaction time limit, as illustrated in Fig. 1. The resonance of interest is between two ground states $|1\rangle$ and $|2\rangle$ coupled by an electromagnetically induced transparency (EIT) [12] or a coherent population trapping (CPT) [13] configuration, with lasers $E_{1}$ and $E_{2}$ under the same frequency modulation (FM). As the two-photon detuning $\Delta$ varies from zero to nonzero, the cross correlation $g^{(2)}(0)$ (defined below) between converted intensity (amplitude) modulations (AM) [14] in $E_{1}$ and $E_{2}$ exhibits a sharp transition from correlation to anticorrelation, with a linewidth far below the CPT zero-power linewidth (determined by interaction time and various decay rates). The physics is as follows. For $\Delta=0$, the transmission spectra of $E_{1}$ and $E_{2}$ overlap and their converted AM are correlated. For $\Delta \neq 0$, the two spectra shift to opposite directions due to the sharp dispersion of EIT. This effectively induces an offset between the two spectra minima much greater than $\Delta$ (offset without ground-state coherence) and creates a considerable range around laser detuning $\delta=0$ that shows opposite slopes responsible for anticorrelations. Therefore, the narrow $g^{(2)}(0)$ width is enabled by ground-state coherence and controlled by laser frequency modulation parameters, as verified in our theory and experiment, which are presented below. Such correlations were previously studied in EIT with noisy diode lasers [15-17] but without identification of the subtransit width.

The dynamics of the generic three-level $\Lambda$ system is described by the Hamiltonian

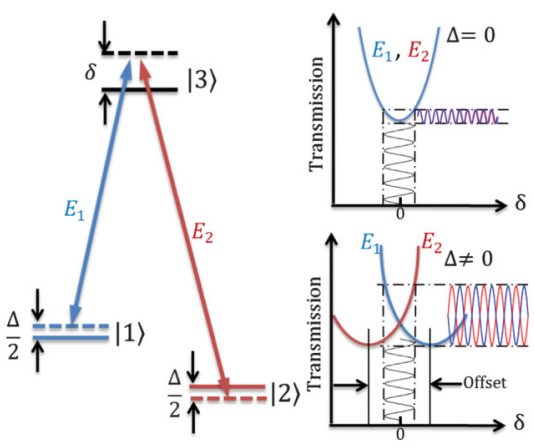

FIG. 1 (color). Principle of the sub-transit-width resonance, shown between $|1\rangle$ and $|2\rangle$ in a three-level $\Lambda$ configuration. $\delta$ is the averaged one-photon detuning, and $\Delta$ is the two-photon detuning, generated by shifting the energy levels with a magnetic field via a Zeeman shift (as shown) or by varying the frequency difference of $E_{1}$ and $E_{2}$. (See the text for details.) 


$$
\begin{aligned}
H= & \Omega_{1}|3\rangle\left\langle 1\left|+\Omega_{2}\right| 3\right\rangle\langle 2|-\delta| 3\rangle\langle 3| \\
& -\frac{\Delta}{2}(|1\rangle\langle 1|-| 2\rangle\langle 2|)+\text { H.c., }
\end{aligned}
$$

where $\Omega_{1,2}$ are Rabi frequencies of $E_{1}$ and $E_{2}$ and H.c. represents the Hermitian conjugate. Here, $\Omega_{1,2}=$ $\Omega_{r_{1,2}} e^{i \lambda \sin \nu t}$, with $\nu$ and $\lambda$ the modulation frequency and modulation depth, respectively. The phenomenological transverse and longitudinal relaxation rates between ground states are $\gamma_{2}$ and $\gamma_{1}$, both including transit and decay effects $[13,18]$. In particular, when transit broadening dominates, the (average) transit time is an effective coherence lifetime [19]. The decay rate of $|3\rangle$ is $\Gamma(\gg \Delta)$. The zero time lag intensity cross correlation between $E_{1}$ and $E_{2}$ is defined by $g^{(2)}(0) \equiv\left\langle\delta I_{1}(t) \delta I_{2}(t)\right\rangle / \sqrt{\left\langle\left(\delta I_{1}\right)^{2}\right\rangle\left\langle\left(\delta I_{2}\right)^{2}\right\rangle}$, where $\delta I_{1,2}$ denote intensity fluctuations of the two transmitted fields after atoms. The positivity (negativity) of $g^{(2)}(0)$ means that two output fields are correlated (anticorrelated). For an optically thin medium, $g^{(2)}(0)$ can be expressed as $g^{(2)}(0)=$ $\left.\left\langle\delta \rho_{31}^{i} \delta \rho_{32}^{i}\right)\right\rangle / \sqrt{\left\langle\left(\delta \rho_{31}^{i}\right)^{2}\right\rangle\left\langle\left(\delta \rho_{32}^{i}\right)^{2}\right\rangle} \quad$ [16], where angled brackets represent a time average and $\delta \rho_{31}^{i}$ and $\delta \rho_{32}^{i}$ are the imaginary parts of fluctuations away from the steady states of the atomic density matrix elements.

To gain physical insights, we pursued analytical solutions in both adiabatic and nonadiabatic regimes by assuming $\Omega_{r 1, r 2}=\Omega$ and equal decay rates from $|3\rangle$ to $|1\rangle$ and $|2\rangle$ [20]. When the modulation frequency $\nu$ is much lower than the optical pumping rate and the modulation amplitude $\lambda \nu$ is much smaller than $\Gamma$, atoms adiabatically follow the steady-state solution

$$
\begin{aligned}
& \rho_{13}^{i}=\frac{-2 \Omega}{\Gamma\left(1+\delta_{0}^{2}\right)}\left[\left(\frac{1}{2}+\rho_{12}^{r}\right)+\delta_{0}\left(1-\frac{2 \Gamma_{p}}{\gamma_{1}+2 \Gamma_{p}}\right) \rho_{12}^{i}\right], \\
& \rho_{23}^{i}=\frac{-2 \Omega}{\Gamma\left(1+\delta_{0}^{2}\right)}\left[\left(\frac{1}{2}+\rho_{12}^{r}\right)-\delta_{0}\left(1-\frac{2 \Gamma_{p}}{\gamma_{1}+2 \Gamma_{p}}\right) \rho_{12}^{i}\right],
\end{aligned}
$$

where $\delta_{0}=\delta /(\Gamma / 2)=\lambda \nu \cos \nu t /(\Gamma / 2)$ is the normalized one-photon detuning, $\Gamma_{p}=\frac{2 \Omega^{2}}{\Gamma\left(1+\delta_{0}^{2}\right)}$ is the reduced optical pumping rate, and $\rho_{12}^{i}$ and $\rho_{12}^{r}$ are the imaginary and real parts of the ground-state coherence $\rho_{12}=\frac{-\Gamma_{p}}{\gamma_{2}+2 \Gamma_{p}+i \Delta}$. The physics now becomes clear. As seen from Eq. (2), the first (second) two terms in the square brackets correspond to the correlated (anticorrelated) intensity. A static offset between the minima of the transmission spectra of $E_{1}$ and $E_{2}$ arises from the anticorrelation terms. For $\delta_{0} \ll 1$, by Taylor expansion and keeping the first and second orders of $\delta_{0}$ in Eq. (2), we find that the correlation (anticorrelation) terms only contain even (odd) orders of $\delta_{0}$. This is because the two transmission spectra have quadratic and parallel FM-AM slopes for $\Delta=0$ and have linear but opposite slopes for $\Delta \neq 0$. Due to the $\rho_{12}^{i}$ factor, the anticorrelation terms are zero at $\Delta=0$ and then grow rapidly with increasing $|\Delta|$. When $|\Delta|=\Gamma_{g 2}$, anticorrelation and correlation terms cancel and $g^{(2)}(0)=0$. Here, $\Gamma_{g 2}$ is the HWHM of $g^{(2)}(0)$ that takes a simple form when $\gamma_{1}=\gamma_{2}$ and $\gamma_{2} \ll 2 \Gamma_{p}$ :

$$
\Gamma_{g 2}=\frac{\gamma_{2}}{\Gamma /(\lambda \nu)}\left(1+\frac{\gamma_{2}}{2 \Gamma_{p}^{0}}\right),
$$

with $\Gamma_{p}^{0}=\frac{2 \Omega^{2}}{\Gamma}$. Equation (3) clearly shows that $g^{(2)}(0)$ has a linewidth below $\gamma_{2}$, and it increases with increasing modulation depth and frequency.

In the nonadiabatic regime, modulation frequency can be much larger than the optical pumping rate and even $\Gamma$. In this case, ground-state coherence cannot follow the laser $\mathrm{FM}$, but the slopes of the transmission spectra of $E_{1}$ and $E_{2}$ around $\delta_{0}=0$ remain parallel for $\Delta=0$ and opposite for $\Delta \neq 0$. We can obtain the first- and second-order responses of the atomic coherence using perturbation theory when the modulation depth $\lambda$ is small:

$$
\begin{aligned}
& \rho_{13}^{i(1)}=-\rho_{23}^{i(1)}=-\frac{2 \Omega J_{1}(\lambda)}{\sqrt{(\Gamma / 2)^{2}+\nu^{2}}} \rho_{12}^{i(0)}, \\
& \rho_{13}^{i(2)}=\rho_{23}^{i(2)}=\frac{2 \Omega J_{2}(\lambda)}{\sqrt{(\Gamma / 2)^{2}+(2 \nu)^{2}}}\left(\frac{1}{2}+\rho_{12}^{r(0)}\right),
\end{aligned}
$$

where $\rho_{i j}^{(n)}$ are the amplitudes of the $n$ th-order responses. Similarly to the adiabatic case, even-order (odd-order) terms correspond to correlation (anticorrelation). The HWHM of $g^{(2)}(0)$ can be derived as

$$
\Gamma_{g 2}=\frac{\gamma_{2}}{C}\left(1+\frac{\gamma_{2}}{2 \Gamma_{p}^{0}}\right)
$$

where $C=\frac{J_{1}(\lambda)}{J_{2}(\lambda)} \sqrt{\frac{(2 \nu)^{2}+(\Gamma / 2)^{2}}{(\nu)^{2}+(\Gamma / 2)^{2}}}$ and $J_{l}$ are the Bessel functions of the first kind. The linewidth is below $\gamma_{2}$, provided that $C>1$, which always holds for relatively small modulation depth.

We performed a proof-of-principle experiment using a ${ }^{87} \mathrm{Rb}$ enriched vacuum vapor cell, with the $C P T$ resonance linewidth limit $\gamma_{2}$ dominated by transit broadening. The Zeeman sublevels of $5^{2} S_{1 / 2}, F=2$ and $5^{2} P_{1 / 2}, F=1$ are ground and excited states, respectively. We used a narrowlinewidth $(<1 \mathrm{MHz})$ diode laser, frequency modulated via either piezoelectric transducer (PZT) or current. Within a three-layer magnetic shield, the cell was inside a solenoid, providing a homogeneous magnetic field for a two-photon detuning adjustment. The cell temperature was at $52{ }^{\circ} \mathrm{C}$. The left and right circularly polarized components of a linearly polarized light entering the cell played the role of $E_{1}$ and $E_{2}$, which were then separately detected after the cell by amplified photodetectors. The ac signals were recorded by an oscilloscope, and the cross correlation of intensity fluctuations was computed off-line. 


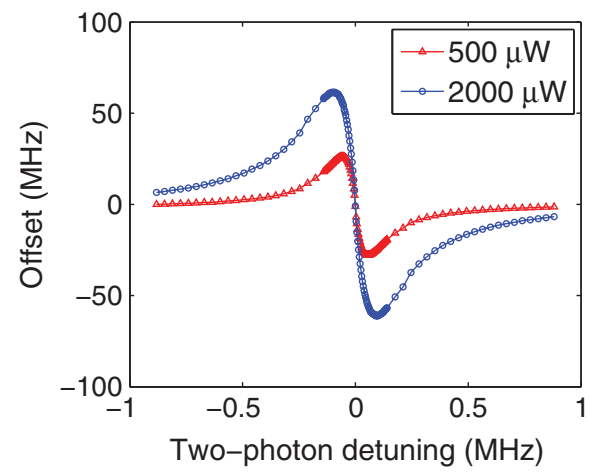

FIG. 2 (color). Measured offset between the transmission minima of $E_{1}$ and $E_{2}$ vs $\Delta$ for two input laser powers. Lines are to guide the eye.

We first measured the offset between the transmission spectra minima of $E_{1}$ and $E_{2}$ vs $\Delta$ (Fig. 2). As expected, the two spectra overlap at $\Delta=0$ and shift to opposite directions for nonzero $\Delta$ (see Fig. 1). The offset value was then retrieved by fitting the spectra with a double Gaussian profile to account for the other excited state. As predicted by Eq. (2), the offset vs $\Delta$ curve is dispersive, and the offset increases with laser power and then saturates. For $2 \mathrm{~mW}$ input power, a maximal offset of $60 \mathrm{MHz}$ was obtained at $\Delta=0.1 \mathrm{MHz}$, corresponding to an effective amplification factor of 600 .

Next, we looked at the $g^{(2)}(0)$ vs $\Delta$ spectrum in the adiabatic regime. The laser frequency was modulated by alternating the PZT voltage at $10.3 \mathrm{kHz}$, with the FM amplitude within tens of MHz. For $\Delta=0$, we observed correlated intensity fluctuations that only have the second harmonic of the modulation frequency [Fig. 3(a)]. At a small nonzero $\Delta$, the correlation can become completely negative and only the first harmonic appears [Fig. 3(b)]. The amplitude of the converted AM is small at zero $\Delta$ and much larger at nonzero $\Delta$ because the FM-AM slopes are quadratic at $\Delta=0$ and linear as $\Delta \neq 0$. Figure 3(c) gives an example of a $g^{(2)}(0)$ spectrum with a linewidth of $2.4 \mathrm{kHz}$. This is about $1 / 30$ of the zero-power $C P T$ width of $75 \mathrm{kHz}$, extrapolated from the measured $C P T$ width vs power curve and consistent with the transit broadening estimated from the $1 / e^{2}$ laser beam diameter of $2.2 \mathrm{~mm}$.

The dependence of the $g^{(2)}(0)$ linewidth upon the laser power and modulation depth was also studied. As shown in Fig. 4(a), the linewidth is larger at lower laser power, consistent with theoretical prediction (dashed line). The observed width increase at higher laser power is due to influences of the residual amplitude modulation (RAM) in the laser. Taking this RAM into account, our simulation reproduces the experimental observation [solid line in Fig. 4(a)]. As the field intensities become more imbalanced, we found that the $g^{(2)}(0)$ width has only a small increase, consistent with the numerical simulation [see the dashed line in Fig. 4(b)]. This allows us to optimize the field intensity ratio against other detrimental effects such
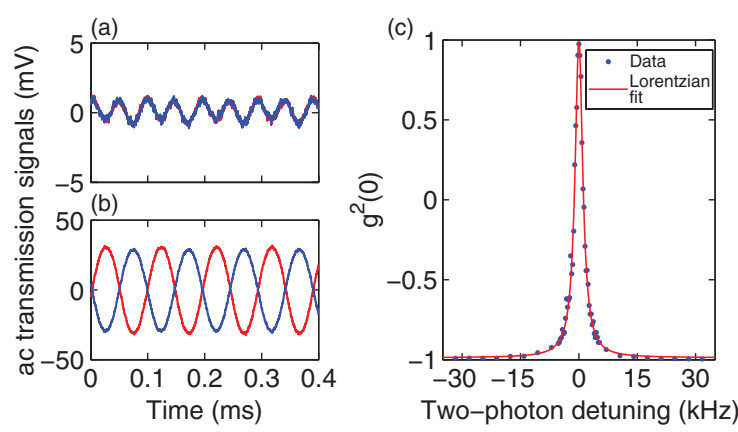

FIG. 3 (color). ac transmitted signals of the two CPT fields (blue and red) for (a) $\Delta=0$ and (b) $\Delta=15 \mathrm{kHz}$. (c) An example of a $g^{(2)}(0)$ spectrum with FWHM $2.4 \mathrm{kHz}$, about $1 / 30$ of the transit width $\gamma_{2} / \pi=75 \mathrm{kHz}$. The input laser power was $300 \mu \mathrm{W}$.

as light shifts in precision measurements. To check the dependence of the $g^{(2)}(0)$ linewidth on modulation depth, we tuned the PZT modulation voltage and observed the linear dependence of the width [Fig. 4(c)], which agrees with the trend seen from Eq. (3). The physics is that a larger range of frequency variation "sees" more parallel slopes and thus reduces anticorrelation. For higher laser power, since the offset is larger (see Fig. 2), the $g^{(2)}(0)$ width is more "immune" to a modulation depth increase.

Finally, we measured the $g^{(2)}(0)$ width in the nonadiabatic regime by current modulating a distributed feedback laser (2 $\mathrm{MHz}$ width). A sub-transit-limited linewidth was also observed (see Fig. 5). The dependence of the $g^{(2)}(0)$
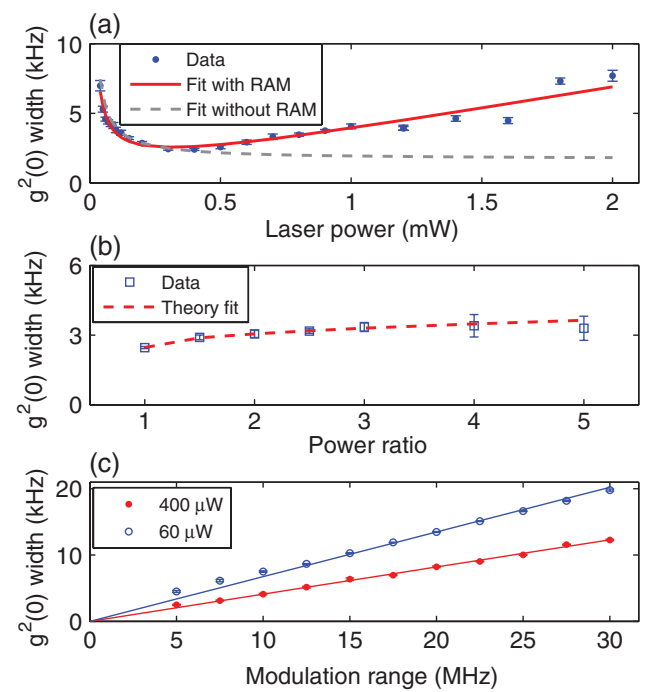

FIG. 4 (color). Dependence of the $g^{(2)}(0)$ FWHM on (a) input laser power, (b) the power ratio of the two $C P T$ beams for a laser power of $300 \mu \mathrm{W}$, and (c) the modulation range for two different laser powers. The full modulation range $2 \lambda \nu$ is $5 \mathrm{MHz}$ for (a), (b). The lines in (c) are linear fits. In (a), (b), an overall scaling factor was applied to the linewidth in the fitting to account for broadening from optical depth and other noises. The transit width is $75 \mathrm{kHz}$. 


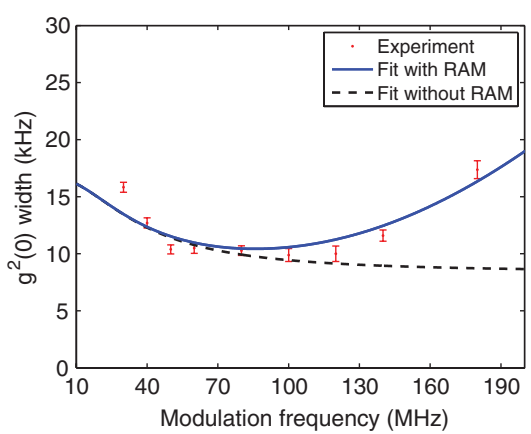

FIG. 5 (color). Measured $g^{(2)}(0)$ FWHM vs modulation frequency in the nonadiabatic regime. The laser power is $800 \mu \mathrm{W}$, and the transit width is $\gamma_{2} / \pi=63 \mathrm{kHz}$.

linewidth on input laser power as well as modulation depth (not shown) follows the same trend as in the adiabatic case. For a small modulation depth $\lambda=0.2$ and modulation frequency from 30 to $180 \mathrm{MHz}$, the $g^{(2)}(0)$ width has a small variation in consistency with Eq. (4). The width increase at higher frequency is due to a combination of the increasing influence of RAM [for the signal is weaker at higher frequency according to Eq. (4)] and slightly larger RAM, as we found in the laser at higher modulation frequency. Calculation taking into account these facts and an overall scaling factor on the linewidth to include broadening from optical depth and other noises fits the data well. An ability to work at both low and high FM frequencies is desirable for practical applications. For example, in $C P T$ clock applications, the laser FM frequency can be chosen to be much higher than rf modulation, which makes the two modulations compatible.

The smallest attainable $g^{(2)}(0)$ width in practice is limited by noises. When the modulation depth is so small that the converted AM signal becomes comparable to noises such as laser intensity noise, RAM, electronics noise, and eventually laser shot noise, the width starts to increase. In our transit-broadened system, we have achieved a minimal linewidth of $1.9 \mathrm{kHz}$ with a signal-to-noise ratio (SNR) [21] of 62 for an average time of $16 \mathrm{~ms}$; when optimized for the ratio of width to SNR, CPT has a linewidth of $166 \mathrm{kHz}$ with a SNR of 1500 . The factor of 3.6 improvement in resolving power is mainly due to the distinct nature of the $g^{(2)}(0)$ observable. As is well recognized, laser frequency noise induces amplitude noise and is a major noise source in precision spectroscopy, including $C P T$-based applications $[13,22]$. However, the $g^{(2)}(0)$ measurement is much less sensitive to noise since only the noise locked to the laser modulation frequency is detected. An enlarged advantage of this method over traditional ones on resolving power is expected with improved FM techniques [23]. In general, narrower resonance is at the price of a lower SNR, but a linewidth considerably narrower than traditional ones is desirable because unknown systematics in resonance line shapes make high ratio line splitting formidable [5]. Therefore, a large line narrowing factor combined with resolving power enhancement is the merit of this technique. In addition, we have numerically examined the possibility to sharpen multipeaks and find that, when $C P T$ fails to completely separate two closely spaced peaks at its resolution limit, this method can clearly resolve them.

Creating quantum correlations between two bright optical fields and also among atomic spins using CPT is currently of great interest [24,25]. Our work paves the way for the realization of such proposals, since inevitable laser frequency noise induces classical correlations in the same way as studied here [26] and understanding its behavior is necessary for achieving quantum correlations. Furthermore, the sharp transition from correlation to anticorrelation observed here has a close resemblance to the behavior of quantum correlations predicted in Ref. [27], and both result from the coherence induced sharp dispersion.

In conclusion, we have demonstrated a conceptually new way to obtain a resonance linewidth far below the transit limit. The proposed scheme can be used to realize a sub-coherence-lifetime-limited linewidth [28]. Our methodology might also be suitable for other level structures, provided that an observable is identified that is only sensitive to phase information near a resonance center. A narrower linewidth combined with improved frequency sensitivity is useful for spectroscopy, precision measurements such as frequency standards [29,30], magnetometry [22], and sensing based on resonance center location. Furthermore, the ability to sharpen multipeaks enables its application in resonance-based imaging [31], where resonance with narrow width and high contrast leads to better resolution. These applications should be implementable in a wide variety of physical systems such as atoms [29], molecules [32], quantum dots [33], diamonds [34], and doped crystals $[35,36]$, etc., where Raman and $C P T$ processes exist.

We thank Vladan Vuletić, Eugeniy Mikhailov, Saijun $\mathrm{Wu}$, Xiangming $\mathrm{Hu}$, Shengwang Du, Michael Hohensee, Irina Novikova, Shiyao Zhu, Weitao Liu, and Mengzhen Zhang for stimulating discussions. L. F. and P. L. contributed equally to this work. This work was funded by the NBRPC (973 Program Grants No. 2011CB921604 and No. 2012CB921604), the NNSFC (Grant No. 10904018), and Fudan University (Y.X.); by the Sherman Fairchild Foundation and the NBRPC (973 Program Grants No. 2011CBA00300 and No. 2011CBA00301) (L. J.); and by the AI-TF New Faculty Grant and the NSERC Discovery Grant (J. W.).

*To whom all correspondence should be addressed. yxiao@fudan.edu.cn

[1] W. Demtröder, Laser Spectroscopy (Springer-Verlag, Berlin, 1998).

[2] W. Gawlik, J. Kowalski, F. Träger, and M. Vollmer, Phys. Rev. Lett. 48, 871 (1982). 
[3] J. F. Lam, D. G. Steel, and R. A. McFarlane, Phys. Rev. Lett. 56, 1679 (1986).

[4] D. J. Gauthier, Y. Zhu, and T. W. Mossberg, Phys. Rev. Lett. 66, 2460 (1991).

[5] H. Metcalf and W. Phillips, Opt. Lett. 5, 540 (1980), and references therein.

[6] P. L. Knight and P. E. Coleman, J. Phys. B 13, 4345 (1980), and references therein.

[7] F. Shimizu, K. Umezu, and H. Takuma, Phys. Rev. Lett. 47, 825 (1981).

[8] H. W. Lee, P. Meystre, and M. O. Scully, Phys. Rev. A 24, 1914 (1981).

[9] M. A. Dugan and A. C. Albrecht, Phys. Rev. A 43, 3877 (1991); M. A. Dugan and A. C. Albrecht, Phys. Rev. A 43, 3922 (1991).

[10] J. Hald, J. C. Petersen, and J. Henningsen, Phys. Rev. Lett. 98, 213902 (2007).

[11] A. Godone, F. Levi, and S. Micalizio, Phys. Rev. A, 65, 031804(R) (2002).

[12] M. Fleischhauer, A. Imamoglu, and J. P. Marangos, Rev. Mod. Phys. 77, 633 (2005).

[13] J. Vanier, Appl. Phys. B 81, 421 (2005).

[14] J. C. Camparo and J. G. Coffer, Phys. Rev. A 59, 728 (1999).

[15] L. S. Cruz, D. Felinto, J. G. Aguirre Gomez, M. Martinelli, P. Valente, A. Lezama, and P. Nussenzveig, Eur. Phys. J. D 41, 531 (2006).

[16] V. A. Sautenkov, Y. V. Rostovtsev, and M. O. Scully, Phys. Rev. A 72, 065801 (2005).

[17] Y. Xiao, T. Wang, M. Baryakhtar, M. Van Camp, M. Crescimanno, M. Hohensee, L. Jiang, D. F. Phillips, M. D. Lukin, S.F. Yelin, and R.L. Walsworth, Phys. Rev. A 80, 041805(R) (2009).

[18] M. Erhard and H. Helm, Phys. Rev. A 63, 043813 (2001).

[19] S. Pustelny, D. F. Jackson Kimball, S. M. Rochester, V. V. Yashchuk, and D. Budker, Phys. Rev. A 74, 063406 (2006).

[20] Without these assumptions, our numerical simulations also confirmed a linewidth below $\gamma_{2}$.

[21] For relevance in calculating resolving power, the SNR is defined as the amplitude of the resonance divided by noise measured at detuning equal to the half-width of the resonance at half-maximum.

[22] P.D. D. Schwindt, S. Knappe, V. Shah, L. Hollberg, J. Kitching, L.-A. Liew, and J. Moreland, Appl. Phys. Lett. 85, 6409 (2004).

[23] M. Gehrtz, G. C. Bjorklund, and E. Whittaker, J. Opt. Soc. Am. B 2, 1510 (1985).

[24] A. Dantan, J. Cviklinski, E. Giacobino, and M. Pinard, Phys. Rev. Lett. 97, 023605 (2006).

[25] A. Sinatra, Phys. Rev. Lett. 97, 253601 (2006).

[26] L. Feng, P. Li, T. Wang, and Y. Xiao (to be published).

[27] F. Wang, X. Hu, W. Shi, and Y. Zhu, Phys. Rev. A 81, 033836 (2010).

[28] In a paraffin-coated vapor cell, we obtained a $g^{(2)}(0)$ FWHM of $12 \mathrm{~Hz}$, about $1 / 3$ of the zero-power EIT FWHM of $35 \mathrm{~Hz}$, limited by the dephasing of the ground-state coherence by the residual magnetic field inhomogeneity within the shields. Further narrowing is possible by optimization of the experiment.

[29] R. Santra, E. Arimondo, T. Ido, C. H. Greene, and J. Ye, Phys. Rev. Lett. 94, 173002 (2005).

[30] T. Zanon, S. Guerandel, E. de Clercq, D. Holleville, N. Dimarcq, and A. Clairon, Phys. Rev. Lett. 94, 193002 (2005).

[31] D. D. Yavuz and N. A. Proite, Phys. Rev. A 76, 041802(R) (2007).

[32] A. Lazoudis, T. Kirova, E. H. Ahmed, L. Li, J. Qi, and A. M. Lyyra, Phys. Rev. A 82, 023812 (2010).

[33] X. Xu, B. Sun, P. R. Berman, D. G. Steel, A. S. Bracker, D. Gammon, and L. J. Sham, Nat. Phys. 4, 692 (2008).

[34] C. Santori, P. Tamarat, P. Neumann, J. Wrachtrup, D. Fattal, R. G. Beausoleil, J. Rabeau, P. Olivero, A. D. Greentree, S. Prawer, F. Jelezko, and P. Hemmer, Phys. Rev. Lett. 97, 247401 (2006).

[35] E. Baldit, K. Bencheikh, P. Monnier, S. Briaudeau, J. A. Levenson, V. Crozatier, I. Lorgere, F. Bretenaker, J. L. Le Gouet, O. Guillot-Noel, and Ph. Goldner, Phys. Rev. B 81, 144303 (2010).

[36] B.S. Ham, P. R. Hemmer, and M.S. Shahriar, Opt. Commun. 144, 227 (1997). 